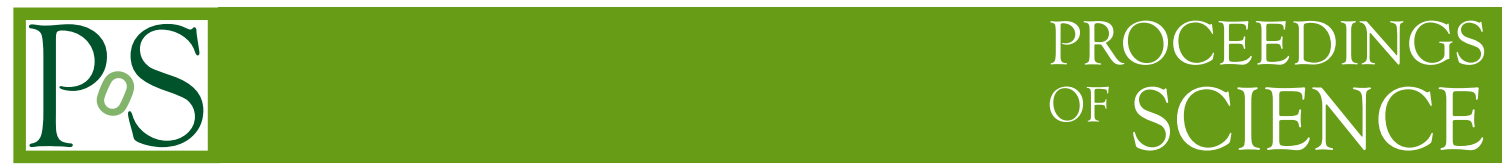

\title{
Fundamental Physics with Slow Neutrons
}

\author{
Hirohiko M. Shimizu*† \\ Furocho, Chikusa, Nagoya 464-8602, Japan, Department of Physics, Nagoya University \\ E-mail: hirohiko.shimizudnagova-u. ip
}

A neutron beamline for the study of the fundamental physics has been constructed at the spallation neutron source of the Japan Proton Accelerator Research Complex (J-PARC). In-flight measurement of the neutron lifetime and the preparation for the study of T-violation using neutrons is in progress. Present status of the beamline and present activities are reported.

KMI International Symposium 2013 on "Quest for the Origin of Particles and the Universe", 11-13 December, 2013

Nagoya University, Japan

\footnotetext{
*Speaker.

${ }^{\dagger}$ on behalf of the NOP collaboration
} 


\section{Introduction}

Neutron is a neutral hadron with the lifetime in the macroscopic range. Large number of neutrons can be decelerated down to the cold region such as $10 \mathrm{meV}$ or less. Such slow neutrons can be applied in the precision measurement of the small influence of physics beyond the standard model of elementary particles. The instantaneously luminous cold neutrons from the spallation neutron source at the Japan Proton Accelerator Research Complex (J-PARC) enables us to carry out new types of high precision measurements. The beam port BL05 of the Material and Life-science research Facility (MLF) of the J-PARC accommodates the neutron beamline "Neutron Optics and Physics (NOP)" [四. The cold neutrons from the coupled moderator are transported to instruments through supermirror guides and benders. A supermirror is a multilayer of two different materials stacked on a substrate, which provides additional reflectivity beyond the critical angle of the total reflection of the material due to the neutron interference in the multilayer. The configuration of

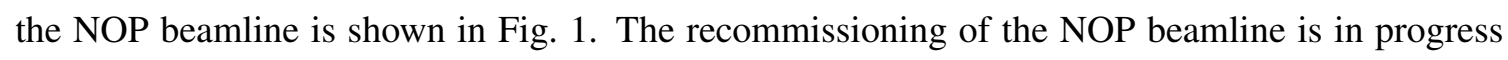

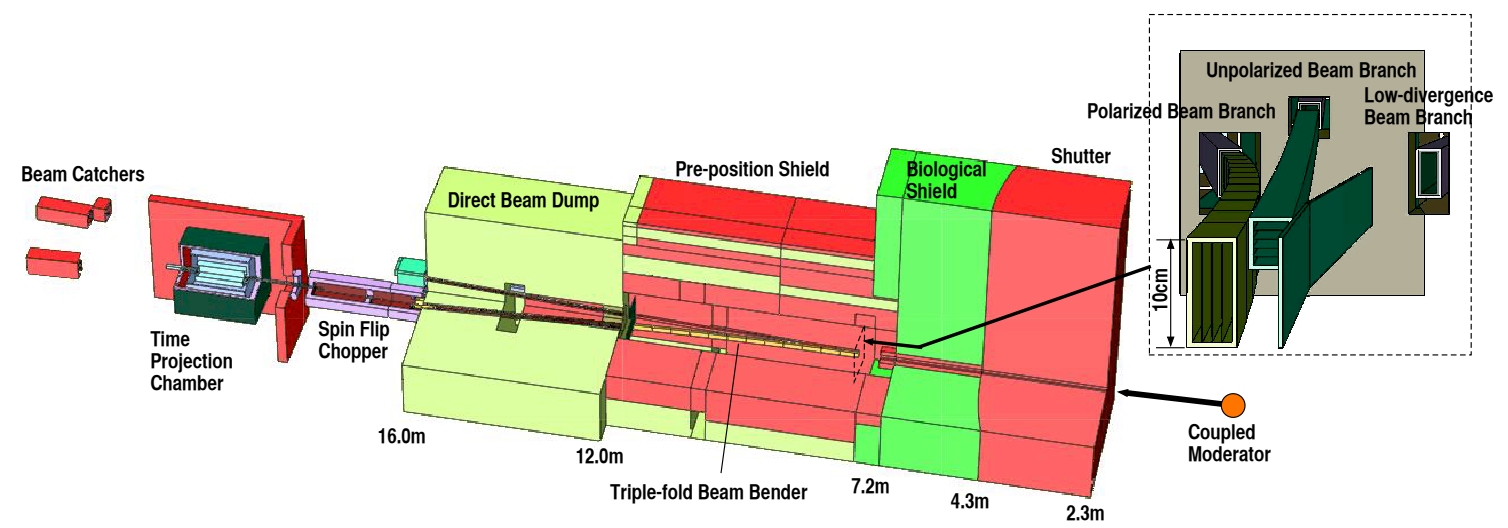

Figure 1: Schematic view of the configuration of the NOP beamline for the study of neutron optics and fundamental physics at the beam port BL05 [0].

following the recovery of the beam delivery after the shutdowns of the J-PARC accelerator in 2011 and 2013. We describe the NOP beam line and introduce the experimental activities.

\section{Neutron Lifetime}

The neutron lifetime $\tau_{n}$ is an input parameter to the primordial nucleosynthesis. The accuracy $\Delta \tau_{n} \sim 1 \mathrm{~s}$ or better is desired for refining the consistency with the observation of the anisotropy of the cosmic microwave background [B]].

The best accuracy was achieved in the measurement of the decrease rate of ultracold neutrons gravitationally confined in a bottle with almost prefect reflectivity [四]. The accuracy was improved by suppressing the neutron leakage due to the up-scattering of neutrons on the inner surface of the bottle by lowering the bottle temperature. The improved value largely deviated from other experimental values and the world average has been changed from $\tau_{n}=(885.7 \pm 0.8) \mathrm{s}$ [可] to $\tau_{n}=(880.1 \pm 1.1) \mathrm{s}$ [目]. The systematic error of this type of experiments is dominated by the 
uncertainty in the correction of the imperfect confinement. A new confinement method using the combination of the inhomogeneous magnetic field and the gravity is being developed to avoid the complexity of the total reflection on material surfaces [ [0].

On the other hand, the $\tau_{n}$ has been also obtained by measuring the decay rate of cold neutrons in flight. The best accuracy in the in-flight geometry was achieved by counting the number of protons accumulated in a Penning-trap through which the cold neutron beam was transmitting [ []]. The result was $\tau_{n}=(886.3 \pm 1.2[\mathrm{stat}] \pm 3.2[\mathrm{syst}]) \mathrm{s}$, which is still deviated from the results of confined method. More measurements using different methods are awaited to clarify the value and the experimental accuracy of neutron lifetime.

Another type of in-beam experiment was last reported in 1989 [眐. Both the production rate of electrons from the $\beta$-decay of neutrons and that of neutron-induced protons via ${ }^{3} \mathrm{He}(n, p)$ were measured by a time-projection chamber (TPC) in which ${ }^{3} \mathrm{He}$ gas was diluted. Assuming the cross section of ${ }^{3} \mathrm{He}(n, p)$ reaction is proportional to the neutron velocity, the lifetime was obtained as

$$
\tau_{n}=\frac{1}{\rho\left({ }^{3} \mathrm{He}\right) \sigma_{0} v_{0}} \times \frac{S_{n} / \varepsilon_{n}}{S_{\beta} / \varepsilon_{\beta}}
$$

where $\rho\left({ }^{3} \mathrm{He}\right)$ is the number density of ${ }^{3} \mathrm{He}$ atoms, $\sigma_{0}$ the absorption cross section via the ${ }^{3} \mathrm{He}(n, p)$ reaction for neutrons with the velocity of $v_{0}, S_{n}$ and $S_{\beta}$ the counting rates of neutron-induced protons and neutron-decay electrons and $\varepsilon_{n}$ and $\varepsilon_{\beta}$ the detection efficiencies of them, respectively. Monochromatic neutron bunches were introduced to the TPC for the suppression of neutroninduced background radiations. However, the number of introduced neutrons was strongly limited because the monochromatic neutron bunches were produced by the combination of the mechanical chopper and the crystal diffraction applied to the reactor-based continuous neutron beam. Consequently, the accuracy was statistically limited as $\tau_{n}=(878 \pm 27[$ stat $] \pm 14[\mathrm{syst}]) \mathrm{s}$.

The statistics of this type of measurement can be remarkably improved since the simple chopping is sufficient to produce monochromatic neutron bunches from the pulsed neutrons of which velocity is well-resolved as a function of the time-of-flight (TOF). A new type of the beam switching optics using the combination of spin-selective mirrors and spin-flipping electromagnets was developed for the fast chopping of neutrons synchronizing to the neutron TOF [ए]]. A TPC was developed by employing low background polymers [ए]]. We expect that the lifetime measurement will be commissioned in 2014 and the data acquisition will be carried out in 2015 .

\section{CP-violation}

\subsection{Electric Dipole Moment}

In the slow neutron experiment, the CP-violation is studied by searching for T-odd correlation terms, which are equivalent to the $\mathrm{CP}$-violation via $\mathrm{CPT}$ theorem. The electric dipole moment (EDM) of the neutron is most commonly searched for. Its present upper limit $2.9 \times 10^{-26} e \mathrm{~cm}$ (C.L.90\%) was obtained in the precision measurement of the dependence of the spin precession frequency of spin polarized ultracold neutrons confined in a material bottle under an electric field [ए2]. The estimated value in the standard model is at the level of $10^{-31}-10^{-32} e \mathrm{~cm}$, which is orders of magnitude below the present experimental sensitivity. Improvement of the experimental sensitivity 
is strongly desired for the discovery of a clue to new physics beyond the standard model [[13]. The statistical accuracy can be written as

$$
\Delta d_{n}=\frac{\hbar / 2}{\alpha E T \sqrt{N}}
$$

where $E$ is the electric field, $T$ the interaction time between neutrons and the electric field, $N$ the number of neutrons and $\alpha$ the quality factor including neutron spin polarization and experimental completeness. Although the larger confinement volume naturally increases the neutron number $N$, it practically increases systematic errors due to the precision of the controlling the magnetic field, electric field etc. Thus the increase of the neutron density is being attempted by introducing accelerator-based ultracold neutron sources and improved ultracold neutron transport optics. A new type of transport optics for pulsed ultracold neutron source is being developed for focusing the arrival time at remote storage volume by accelerating/decelerating neutrons as a function of neutron time-of-flight [14]].

The interaction time $T$ was about $10^{2} \mathrm{~s}$ and the applied electric field of $E=10^{6} \mathrm{~V} \mathrm{~m}^{-1}$ was chosen to suppress the influence of the magnetic field induced by the leakage current in the material bottle. Recent EDM measurements with confined ultracold neutrons were carried out with the value of $E T \sim 10^{8} \mathrm{~V} \mathrm{~s} \mathrm{~m}^{-1}$.

Historically, the EDM measurement was started in the in-flight geometry [15]]. A possibility to improve the EDM sensitivity of in-flight experiment is being discussed assuming to employ the intense pulsed cold neutrons from spallation sources and improved optics to transport polarized cold neutrons as long as $50 \mathrm{~m}$ or longer [ए]].

Application of the electric field in single crystals is another possibility as studied in 1960s [ए]]. The forward neutron scattering amplitude can be written as

$$
f(\boldsymbol{q})=f_{0}+i \frac{2 e \mu_{n}}{\hbar c}(Z-F(\boldsymbol{q})) \frac{\boldsymbol{\sigma} \cdot(\boldsymbol{k} \times \boldsymbol{q})}{q^{2}}+i \frac{2 m e d_{n}}{\hbar^{2}}(Z-F(\boldsymbol{q})) \frac{\boldsymbol{\sigma} \cdot \boldsymbol{q}}{q},
$$

where $\boldsymbol{q}$ is the momentum transfer, $\boldsymbol{k}$ the incident momentum, $\boldsymbol{\sigma}$ the unit vector parallel to neutron spin, $f_{0}$ the nuclear scattering length, $Z$ the atomic number, $F(\boldsymbol{q})$ the atomic form factor, $\mu_{n}$ the neutron magnetic moment and $d_{n}$ the neutron EDM. The second and third terms corresponds to the magnetic and electric scattering. The neutron spin rotation about the $\boldsymbol{q}$ signals the EDM. Improved sensitivity can be expected if we choose a diffraction of a single crystal which shows a large value

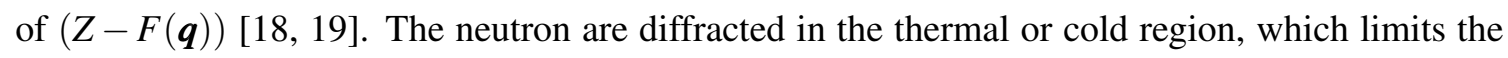
interaction time $T$ at the level of $10^{-3} \mathrm{~s}$. The short interaction time can be compensated by the large effective electric field corresponding to the electric scattering as large as $10^{11} \mathrm{~V} \mathrm{~m}^{-1}$, which results in the value of $E T \sim 10^{8} \mathrm{~V} \mathrm{~s} \mathrm{~m}^{-1}$.

The experimental accuracy of the neutron EDM in the single crystal diffraction is dominated by the quality of the single crystal. The quality measurement is being prepared with the single crystal of bismuth germinate at J-PARC/MLF.

\subsection{T-odd Correlation in Compound Nuclei}

Neutron-induced compound nuclear states are observed as resolved resonances as narrow as $0.1 \mathrm{eV}$ in the total cross section of neutrons incident to nuclei in the incident energy region of about 
$10^{-1}<E_{n}<10^{3} \mathrm{eV}$. Most of the resonances are s-wave resonances with the cross section of about kilobarns and small p-wave resonances are also observed with the cross section of about barns. Large parity-violating effects are observed in the resonance cross section about the helicity of incident neutrons [ $[2]$. The largest helicity dependence amounts to a few percent of the total cross section, which corresponds to the enhancement of $10^{6}$ compared with the helicity dependence in nucleon-nucleon total cross section. The enhancement is explained as the interference between s-wave and p-wave neutron partial waves in the entrance channel to the compound state.

The enhancement mechanism may be applicable to T-violating effect as pointed out in Ref. [2]]]. The forward scattering amplitude of neutrons with spin-polarized nuclear target is given

$$
f=A+B(\boldsymbol{\sigma} \cdot \boldsymbol{I})+C(\boldsymbol{\sigma} \cdot \boldsymbol{k})+D(\boldsymbol{\sigma} \cdot(\boldsymbol{k} \times \boldsymbol{I})),
$$

where $\boldsymbol{I}$ is the spin of the target nucleus. The last term is odd under the time-reversal operation. The behavior of the neutron spin on transmission through a thick target can be described in the neutron optical formalism, in which the final state interaction can be ignored, as

$$
\begin{aligned}
U_{f} & =e^{i \zeta A}\left(\cos b+i \frac{\sin b}{b} \zeta B(\boldsymbol{\sigma} \cdot \boldsymbol{I})+i \frac{\sin b}{b} \zeta C(\boldsymbol{\sigma} \cdot \boldsymbol{k})+i \frac{\sin b}{b} \zeta D(\boldsymbol{\sigma} \cdot(\boldsymbol{k} \times \boldsymbol{I}))\right) U_{i}, \\
b & =\zeta \sqrt{B^{2}+C^{2}+D^{2}} \\
\zeta & =\frac{2 \pi \rho z}{k}
\end{aligned}
$$

where $U_{i}$ and $U_{f}$ are the initial and final spin state, $z$ the spatial coordinate along the beam axis and $\rho$ the number density of target nuclei. Therefore the non-zero value of the T-odd correlation term in finite thickness target unambiguously signals the T-violation.

The T-violation can be enhanced as a result of the interference between partial waves with different channel spins. The comparison of the value of the T-odd correlation term and the EDM value is being discussed based on the effective field theory [22]. Present upper limit of the EDM roughly corresponds to the T-violating cross section of about $10^{-10} \mathrm{~b}$ in effective nucleon interaction, which may be enhanced up to $10^{-4} \mathrm{~b}$ assuming the enhancement of $10^{6}$. The effect is sufficiently large to be detected using the intense epithermal neutrons from the present spallation sources.

The detailed information of the mixing of partial waves in the entrance channel necessary to estimate the T-violation enhancement factor can be obtained in the angular distribution of individual $\gamma$-ray transitions if both the spin of compound state and that of the final state are known [23]]. Measurement of the $\gamma$-ray angular distribution is in progress at the ANNRI (Accurate NeutronNucleus Reaction measurement Instrument) installed at the beam port BL04 of the J-PARC/MLF.

\section{Discussion and Summary}

The improvement of experimental sensitivity using neutrons to new physics beyond the standard model is newly enabled by the combination of the instantaneously luminous pulsed neutron beam and the advances in neutron optics.

One of further possibilities is the search for new forces using neutrons. The scattering cross section of unpolarized neutron by atoms can be described as

$$
\frac{\mathrm{d} \sigma}{\mathrm{d} \Omega} \propto\left|a_{0}+a_{n e} Z F_{e}(\theta)+a_{\mathrm{G}} F_{\mathrm{G}}(\theta)\right|^{2}
$$




$$
\simeq a_{0}^{2}+2 a_{0} a_{n e} Z F_{e}(\theta)+a_{n e}^{2} Z^{2} F_{e}(\theta)^{2}+2 a_{0} a_{\mathrm{G}} F_{\mathrm{G}}(\theta)
$$

where $a_{0}$ is the nuclear scattering length, $a_{n e}$ the electron-neutron scattering length, $F_{e}(\theta)$ the atomic form factor. The $a_{\mathrm{G}}$ represents the scattering length via an unknown weak interaction and $F_{\mathrm{G}}(\theta)$ the form factor. New force beyond the standard model can be searched for the precision measurement of the angular distribution in the scattering cross section if both the nuclear scattering length and the electron-neutron form factor is well known. It can be also searched for by applying the neutron interferometry to measure the phase difference between neutron paths passing through different potentials due to new forces. The technology of multilayer neutron interferometry has been remarkably improved [24]. Modification of the multilayer interferometer is in progress for accepting pulsed neutrons by employing supermirrors to configure the interferometer.

The cold neutron beam delivery is expected to be restarted in 2014. The researches in the neutron fundamental physics will be also restarted soon after the beamline recommissioning.

\section{References}

[1] K. Mishima et al., Nucl. Instrum. Methods Phys. Res. A600 (2009) 342.

[2] Y. Arimoto et al., Prog. Theor. Exp. Phys. (2012) 02B007 DOI: 10.1093/ptep/pts075.

[3] G.J.Mathews, T.Kajino, T.Shima, Phys.Rev. D71 (2005) 021302(R).

[4] A. Serebrov et al, Phys. Lett. B 605 (2005) 72.

[5] S. Eidelman et al., Phys. Lett. B 592 (2004) 1.

[6] J. Beringer et al., Phys. Rev. D86 (2012) 010001.

[7] V. Ezhov. Seventh International Workshop on Cold and Ultracold Neutrons, St. Petersburg Russia, 2009

[8] A. T. Yue et al., Phys. Rev. Lett. 111 (2013) 222501.

[9] R. Kossakowski et al., Nucl. Phys. A 503 (1989) 473.

[10] K.Taketani et al., Nucl. Instrum. Methods Phys. Res. A634 (2011) 134.

[11] H. Otono, dissertation, University of Tokyo (2012).

[12] C. A. Baker et al., Phys. Rev. Lett. 97 (2006) 131801.

[13] M. J. Ramsey-Musolf and S. Su, Phys. Rep. 456 (2008) 1.

[14] Y. Arimoto et al., Phys. Rev. A 86 (2012) 023843.

[15] J. Smith, E. M. Purcell, and N. F. Ramsey, Phys. Rev. 108 (1957) 120.

[16] F. M. Piegsa, Phys. Rev. C88 (2013) 045502.

[17] C. G. Shull and R. Nathans, Phys. Rev. Lett. 19 (1967) 384.

[18] M. Forte and C. M. E. Zeyen, Nucl. Instrum. Methods 284 (1989) 174, C. M. E. Zeyen et al., J. Phys. Soc. Jpn. 65 (1996) Suppl. A pp. 177.

[19] V. V. Federov et al., Physica B406 (2011) 2370.

[20] G. E. Mitchell et al., Phys. Rep. 354 (2001) 157. 
[21] V. P. Gudkov, Phys. Rep. 212 (1992) 77.

[22] Y. -H. Song et al., Phys. Rev. C83 (2011) 065503.

[23] V. V. Flambaum and O. P. Sushkov, Nucl. Phys. A435 (1985) 352.

[24] Y. Seki et al., J. Phys. Soc. Jpn. 79 (2010) 124201. 